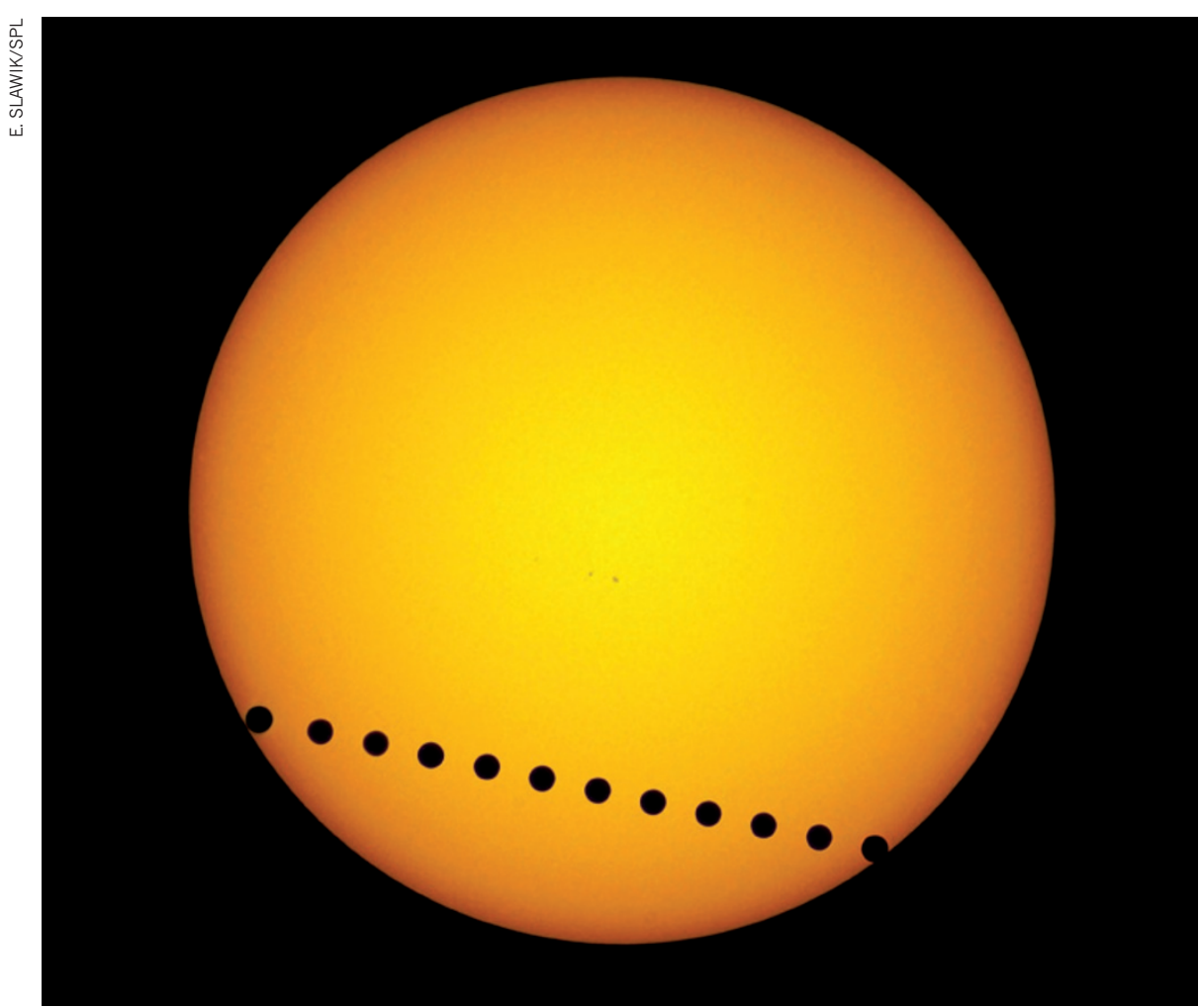

A composite image of the June 2004 transit of Venus as seen from Waldenburg, Germany.

\title{
Last chance to see
}

\section{The June 2012 transit of Venus across the Sun offers an opportunity to check our methods for spotting distant planets crossing far-away stars, says Jay M. Pasachoff.}

$\mathrm{T}$ The sight of Venus silhouetted against the Sun is exceedingly rare. Since 1631, when a transit of Venus was predicted but unobservable from much of Europe (where most early telescopes were located), such transits have been seen only six times: in $1639,1761,1769,1874,1882$ and 2004. Our last chance, until 2117, to see and study such a transit from Earth will be on 5-6 June (http:// transitofvenus.info).

Earlier transits were subject to intense scientific scrutiny. Hundreds of expeditions were sent out from around the world to observe the transits of 1761 and 1769 , in efforts to triangulate the distance from Earth to the Sun. Today, the distance known as the astronomical unit has long been settled by more accurate means. Many people now think of transits within our Solar System only as public-outreach opportunities. Yet there is still much to be learned from modern transits, by taking advantage of new ideas, techniques and scientific capabilities.

For example, my colleagues and I used spacecraft observations of Mercury's 1999 transit to settle a long-standing mystery about the appearance of transiting planets.

Despite modern scientists' ability to send probes to Venus for close-up scrutiny, its transits as seen from Earth (and elsewhere) still provide unique information, and they give us the opportunity to calibrate or improve our methods for finding far-off planets around distant stars.

\section{BLACK DROP}

I became interested in transits a decade ago, when I learned from a historical talk that most contemporary books and articles wrongly attribute the cause of the phenomenon known as the black-drop effect ${ }^{1}$.

In the 1700s, the method for determining the average distance to the Sun depended on the accurate measurement, to about a second, of the period from when Venus fully entered the solar disk to when it began to depart. But such measures were confounded by the appearance of a dark ligature between Venus's silhouette and the space outside the solar disk. This 'black drop' would grow for about a minute and then pop. The effect made the uncertainty in timing much worse than had been hoped for. For centuries, the black-drop effect limited the solution to what was arguably the most important problem in astronomy: the distances of planets from the Sun. Even by the 2000s, most publications still attributed the black-drop effect to the diffraction of light around Venus, the refraction of light by its atmosphere or even an optical illusion - even though the evidence showed these explanations to be false ${ }^{1}$.

Glenn Schneider, an astronomer at the University of Arizona in Tucson, and I looked for a black drop in the data taken for Mercury's 1999 transit by NASA's TRACE satellite. We found one, confirming that neither the planet's nor Earth's atmosphere was needed to produce the phenomenon (although Earth's atmosphere exaggerates the effect when viewed from Earth's surface). Our analysis showed that two effects could fully explain the black drop as seen from space: the inherent blurriness of the image caused by the finite size of the telescope, and an extreme dimming of the Sun's surface just inside its apparent outer edge $e^{2,3}$. Apart from settling a point of historic interest, understanding this effect may yet help to unravel observations of other planets' transits across far-away stars.

My colleagues, my students and I set out to test our ideas about the black-drop effect through observations of the 8 June 2004 transit of Venus. This brought an unrelated surprise. Images taken by TRACE during ingress and egress showed a rim of light that appeared over the trailing side of Venus about 20 minutes before it fully entered the solar disk ${ }^{4}$ - the result of refraction of sunlight towards us by Venus's atmosphere. We were flabbergasted that Venus's atmosphere was so visible (although it turns out that this effect had also been noted during the 1874 transit). The arc of light was asymmetrical - brighter at some latitudes on the planet than at others.

With colleagues, we later compared our results to data from land-based telescopes ${ }^{5}$ and from the European Space Agency's Venus Express probe, which arrived at the planet in 2006 (ref. 4). These comparisons helped to confirm, for example, observations that the planet's haze is at lower altitudes at its poles than at its equator.

The transit showed us some things that the probe could not. For many atmospheric measures, Venus Express sees only one small slice of the atmosphere at a time during sunrise or sunset: a measure is taken at one latitude one day and at another latitude the next, making it impossible to know whether any differences are due to changes in space or to changes in time. Only through 

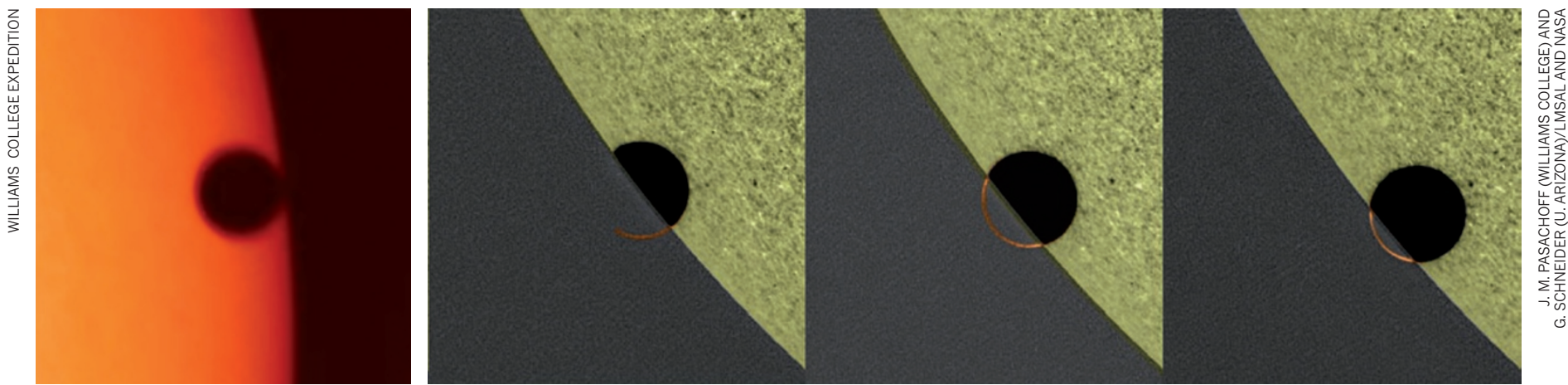

Left: the black-drop effect connects the transiting planet to dark space. Right: Venus's atmosphere seen from space as refracted sunlight, 2004 transit.

transit observations can we see an entire arc of the planet's atmosphere at once. Further comparisons between the two will help to cross-calibrate both techniques.

From that same 2004 transit, we were able to use NASA's ACRIM III instrument on the ACRIMSAT spacecraft to detect not just the $0.1 \%$ drop in light from the Sun during the central part of Venus's passage, but also the smaller changes that resulted when Venus blocked parts of the dim outer regions of the solar disk ${ }^{6}$. We could even calculate the diameter of Venus (which is already known), which should help to gauge the accuracy of this technique in assessing the sizes of exoplanets.

\section{HOME TRUTHS}

The view that ACRIM III and the Total Irradiance Monitor on NASA's SORCE spacecraft will have of Venus's transit across the Sun is analogous to the view that planet-hunting satellites and ground-based telescopes have of exoplanets moving across other stars. NASA's Kepler spacecraft has found more than 2,000 exoplanet candidates so far, but it has confirmed fewer than 100 . One problem in confirming sightings is knowing whether the dip in light seen from a distant star is due to a transiting planet or to some other effect, such as starspots (akin to sunspots).

The upcoming transit offers a particularly good opportunity to study this effect. The 2004 Venus transit happened at a time of minimal solar activity, when no sunspots were visible. By contrast, a 2006 transit of Mercury - whose disk in transit is just 3\% of the area of Venus as seen from Earth - happened during the passage of a large sunspot across the face of the Sun. The dip in the total solar irradiance from that transit was lost amid the noise of solar activity and the limitations of the spacecraft. As expected at this phase of the sunspot cycle, 2012 is turning out to be a year of relatively high solar activity, so we expect the Venus transit to provide a situation that more closely resembles that of 'spotty' stars hosting exoplanets.

It is too soon to know exactly how the study of transits in our Solar System will help us to interpret observations of distant exoplanets,

but transits are so rare that to squander these opportunities would be a crime. We owe it to future astronomers - especially those who will observe the next transit of Venus, in 2117 - to collect as much data as possible. One never knows what will prove vital to future research.

In the eight years since the last Venus transit, the equipment has improved. The TRACE satellite has been replaced by the Solar Dynamics Observatory, which has about the same resolution, but over the entire Sun, giving a full set of filtered images every 10 seconds. A US telescope on Japan's Hinode satellite has even better resolution.

All hands will be on deck to watch the coming transit. Using telescopes on Haleakala in Hawaii, my team will look, in part, at the polarization of sunlight by Venus's atmosphere, which will tell us something about particle size. With colleagues at several institutions in France, we will use a set of nine coronagraphs around the world to study the bright arc of Venus's atmosphere.

In the continen-

"We owe it to the astronomers who will observe the 2117 transit of Venus to collect as much data as possible."

tal United States, our group will observe the transit using a massive spectrograph at the Richard B. Dunn Solar Telescope of the National Solar Observatory at Sacramento Peak in New Mexico. We have purchased a new filter at the wavelength of carbon dioxide - a major constituent of Venus's atmosphere - for these observations. This will provide a unique, detailed spectrographic study of a relatively wellknown atmosphere during a transit, which we can compare to studies of unknown exoplanet atmospheres.

There are some more cunning, indirect ways of watching the transit. The Hubble Space Telescope, which is too sensitive to be pointed directly at the Sun, will be used to observe sunlight reflected from the lunar surface. The low light in this observation mimics what night-time telescopes see when studying exoplanet transits.
We have also applied for time on Hubble to observe other transits, including a 20 September 2012 transit of Venus as seen from sunlight reflected off Jupiter; if granted that time, we will also apply to observe a 5 January 2014 transit of Earth as seen from Jupiter. Many people will be keen to see what a habitable, populated planet looks like in transit, and it would be a sheer delight to watch Earth pass in front of the Sun. The next such opportunity will not be until 2026.

NASA's Cassini spacecraft, now in orbit in the Saturn system, also has an unusual vantage for spying on transits - and it can directly observe the Sun. We have already arranged for it to observe a transit of Venus on 21 December 2012.

In 1874, astronomer Richard Proctor wrote, in his book Transits of Venus, "Let it be hoped that the success of operations conducted by the various scientific nations in 1874 and 1882 may be such that preliminary difficulties will hereafter be remembered only as obstacles successfully removed and in good time." One can only hope that future scientists will look back at the relatively feeble attempts of twenty-first-century astronomers and say that we, too, did the best we could. SEE BOOKS \& ARTS P.305

Jay M. Pasachoff is Field Memorial Professor of Astronomy at Williams College, Williamstown, Massachusetts 02167, USA. He is also chair of the International Astronomical Union's Working Group on Solar Eclipses and vice-chair of the American Astronomical Society's Historical Astronomy Division. e-mail:eclipse@williams.edu

1. Schaefer, B. E. Bull. Am. Astron. Soc. 32, 1383 (2000).

2. Pasachoff, J. M., Schneider, G. \& Golub, L. in Transits of Venus: New Views of the Solar System and Galaxy (ed. Kurtz, D. W.) 242-253 (Cambridge Univ. Press, 2005).

3. Schneider, G., Pasachoff, J. M. \& Golub, L. Icarus 168, 249-256 (2004).

4. Pasachoff, J. M., Schneider, G. \& Widemann, T. Astron. J. 141, 112 (2011).

5. Tanga, P. et al. Icarus 218, 207-219 (2012).

6. Schneider, G., Pasachoff, J. M. \& Willson, R. C. Astrophys. J. 641, 565-571 (2006). 